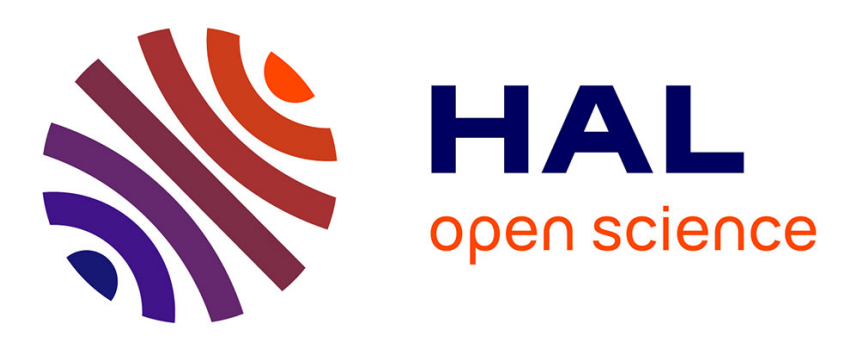

\title{
Flow and Particles Deposition in Rabit and Rat Airways Under Realistic Inflow Rate
}

Yannick Hoarau, Philippe Choquet, Christian Goetz, A. Fouras, S. Dubsky, Marianna Braza, S. Saintlos-Brillac, Franck Plouraboué, D. Lo Jacono

\section{- To cite this version:}

Yannick Hoarau, Philippe Choquet, Christian Goetz, A. Fouras, S. Dubsky, et al.. Flow and Particles Deposition in Rabit and Rat Airways Under Realistic Inflow Rate. ERCOFTAC Symposium on Unsteady Separation in Fluid-Structure Interaction,, Jun 2013, Mykonos, Greece. pp.193-204, 10.1007/978-3-319-27386-0_12 . hal-03146239

\section{HAL Id: hal-03146239 \\ https://hal.science/hal-03146239}

Submitted on 18 Feb 2021

HAL is a multi-disciplinary open access archive for the deposit and dissemination of scientific research documents, whether they are published or not. The documents may come from teaching and research institutions in France or abroad, or from public or private research centers.
L'archive ouverte pluridisciplinaire HAL, est destinée au dépôt et à la diffusion de documents scientifiques de niveau recherche, publiés ou non, émanant des établissements d'enseignement et de recherche français ou étrangers, des laboratoires publics ou privés. 


\title{
Flow and Particles Deposition in Rabit and Rat AiRWAYS UNDER REALISTIC INFLOW RATE
}

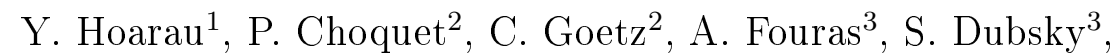 \\ M. Braza ${ }^{4}$, S. Saintlos-Brillac ${ }^{4}$, F. Plouraboué ${ }^{4}$, D. Lo Jacono ${ }^{4}$ \\ ${ }^{1}$ ICUBE, Université de Strasbourg, CNRS \\ ${ }^{2}$ Imagerie Préclinique des Hôpitaux Universitaires de Strasbourg, \\ ${ }^{3}$ Laboratory of Dynamic Imaging, Monash University, Australia, \\ ${ }^{4}$ Institut de Mécanique des Fluides de Toulouse, INPT/CNRS/UPS,
}

\begin{abstract}
The understanding of the flow structures and the particle transport/deposition across the human bronchial system remains a challenge to achieve because of the complexity of the geometry of human lungs. This work relies a strong collaboration between physicians, medical imaging researchers, fluid mechanics researcher and CFD researchers. Four configurations of airways (the generic Weibel model, the Human model proposed by Hiroko Kitaoka, a realistic Rat lung obtained by $\mu$-CT and a realistic rabbit geometry obtained by a synchrotron based CT) have been generated, meshed and simulated using the CFD commercial package CFD-ACE. Both steady and realistic inflow rates have been studied as well as the associated transport and deposition of particles.
\end{abstract}

Key words : particle deposition; rat lungs; rabbit lungs; human lungs; numerical simulations.

\section{Introduction}

The aerosol particles present in our environment are increasingly identified as risk factors for health, but aerosols can also be used for diagnostic or therapeutic purposes to treat lung diseases. In particular, small particles that reach the surface of gas exchange in the alveolar region of lungs are considered most harmful to health. Inhalers, for example, are commonly used to administer drugs [5]. In cases of lung disease, administration of medication by aerosol has the advantage of reaching the region directly affected and requires lower doses (side effects are minimized).

The morphological descriptions of the bronchial tubes of the man appeared in the literature since 1963, when Weibel [20] introduced a physical symmetric model. Horsfield and Cumming [10] have subsequently proposed an anatomical representation of asymmetric lung tubes taking the Weibel model as a basis. Horsfield, Dart and Olson [11] have produced two types of asymmetric mathematical models based on data obtained from measurement of a resin cast of a normal human bronchiol tree. The models permit calculation of physiologic variables to be made while taking asymmetry into account. Hammersley and Olson [8] presented a model of symmetric and asymmetric bifurcations in the human lung adequate for generations 6 to 12. In 2004, Hegedus et al. [9] provided a detailed mathematical description of a 
morphologically realistic airway of first five generations.

The human lung is a sequence of bifurcations in two parts. It has an average of 23 generations of bifurcations, or about 17 million of bifurcations. The last 5 generations are covered with cellular structures through which the exchange takes place with the blood gas [21].

The researchers eventually want to understand the flow structure and particle transport/deposition across the human bronchial system. This goal remain a challenge to achieve because of the complexity of the geometry of human lungs. However, few researchers have managed to simulate the flow in models with many generations. Nowak et al. [19] presented the flow field and simulation results of particle transport to the lung airways obtained from a CT-scan. Comparisons of calculation results for a realistic model and the model of Weibel showed a profile of deposition of micro-particles that had differences over a moderate range of Reynolds numbers of 120-2000 for both steady flow and unsteady. Although a CT-scan provides a more realistic geometry, it can not provide respiratory beyond nine generations, which limits its application to the study of all lung airways. Miki et al. [18] digitized and then calculated an actual configuration to the seventh generation. Ertbruggen et al. [6] described a three-dimensional structure based on the anatomy of the airways of the lung starting from the trachea until the 17th bifurcations to simulate stationary flow with transport of micro-particles. The maximum deposition of particles has been identified on the bifurcation with the highest 'off-plan' angle compared to other tubes of the same generation, thus demonstrating the role of non-flatness on the deposition of particles micrometer size. A mesh structured multi-block was chosen and refined until the maximum difference between the velocity profiles was below $2 \%$.

The deposition of particles takes place, usually, under the action of three mechanisms :

- impact by inertia : this mode of deposition depends on the diameter and the velocity of the particles. Those whose size is greater than $10 \mu \mathrm{m}$ impact in the oropharynx while the smaller worm their way deep into the bronchial tree.

- sedimentation resulting from the effect of gravity, which revealed the particles to the bronchial wall. It affects mainly small particles $(1$ to $5 \mu \mathrm{m})$ and involves only the bronchial tubes and alveoli. It is maximum for low airflow and can be promoted by a simple respiratory pause at the end of inspiration.

- diffusion or Brownian motion is due to the chaotic bustle of small particles (smaller than $0.5 \mu m$ why undergo collisions with gas molecules. This mode of deposition is insignificant as $80 \%$ of the particles remain in suspension and then are removed at the expiration [13].

\section{Methods}

Our study focuses on the numerical modelling of the flow and particles deposition in airways using the commercial package CFD-ACE. The objective of this study is to propose a reliable and complete numerical model of transport of particles in the 
pulmonary flow by taking into account the full breathing cycle. For the moment the movement of the airways imposed by the breathing are not taken into account.

This study is divided into four parts :

- First the CFD-ACE package is validated regarding flow [3, 25] and particles deposition in a simple model [14, 2]

- Simple geometries derived from the Weibel model are studied

- An anatomically realistic model of rat lung is simulated

- A more complex geometry of rabbit lung is simulated

The particles trajectories and deposition are obtained by solving a Lagrangian transport equation where only the drag force is retained. The deposition efficiency is computed as the ratio of the amount of particles sticked at the wall over the total number of released particles.

The rat geometry is obtained after an adult rat killed by IV administration of barbiturates, was infused with a iodine contrast agent $(10 \% \mathrm{v} / \mathrm{v}$, Visipaque320, GE Healthcare, Chalfont St Giles, UK). Imaging of the thorax was performed ex vivo using an X-Ray CT scanner (eXplore Vision 120, GE Healthcare, Waukesha, USA). The protocol used involved 360 views over $360^{\circ}$, with 1 frame averages, at $100 \mathrm{kV}$, $50 \mathrm{~mA}$. After reconstruction using a Feldkamp algorithm of back-projection, the imaging volume was made of cubic voxels of $49 \mathrm{~m} \times 49 \mathrm{~m} \times 49 \mathrm{~m}$. Visualisation was performed using MicroView (GE Healthcare, Waukesha, USA). After inversion of grey levels, a surface rendering (marching cubes algorithm) was applied after threshold. The surface was then exported in STL format and prepared in GMSH before being meshed in CFD-GEOM.

The rabbit geometry was obtained using synchrotron-based dynamic computed tomography. Details of the experimental technique and segmentation protocol are provided elsewhere [4]. Briefly, newborn rabbit pups are ventilated and imaged using propagation-based phase-contrast imaging. The lungs are an ideal sample for phasecontrast imaging, as they exhibit large phase boundaries at air-tissue interfaces. This technology can provide rapid imaging of the lungs without contrast agent [7]. By synchronising the image acquisition with the ventilation and rotating the sample, fourdimensional $(4 \mathrm{D}=3 \mathrm{D}+$ time $)$ images of the bronchial tree can be achieved. Again, one STL geometry was prepared in GMSH before being meshed in CFD-GEOM.

Kitaoka et al [15] has proposed a three-dimensional (3D) model of the human airway tree using a deterministic algorithm that can generate a branching duct system in an organ through the free software Lung 4 Cer (http ://www7b.biglobe.ne.jp/ lung4cer/indexEng.html). We have generated a model with more than 7 bifurcations resulting in more than 300 outlets.

Part of this work was performed during H. Ilmi PhD thesis [12]. 


\section{Governing equation}

\subsection{Airflow equations}

For a laminar incompressible flow, the fluid transport equations can be written as : continuity

$$
\nabla \cdot \vec{v}=0
$$

momentum

$$
\frac{\partial \vec{v}}{\partial t}+(\vec{v} \cdot \nabla) \vec{v}=-\frac{1}{\rho} \nabla p+\nabla \cdot\left[\nu\left(\nabla \vec{v}+(\nabla \vec{v})^{T}\right)\right]
$$

where $\vec{v}$ is the velocity vector, $\mathrm{p}$ the pressure, $\rho$ the fluid density and $\nu$ the fluid kinematic viscosity, ()$^{T}$ represents the transpose of ( ) [3], [22].

\subsection{Particle transport and deposition equations}

In this work, simulations are performed for particles of diameter $d_{p}=5 \mu \mathrm{m}$. In light of the small particle Reynolds numbers, $R e_{p} \sim\left|\vec{v}-\vec{v}_{p}\right| \ll 1$ and a large density ratio, $\rho_{p} \gg \rho$, the drag force is dominant, based on order-of-magnitude argument (Clift, Grace, \& Weber, 1978; Crow, Sommerfeld, \& Tsuji, 1998; Kleinstreuer, 2003). These particles are relatively large, so Brownian motion can be neglected. The particulate material considered is far denser than fluid, causing terms that depend on the density ratio, such as pressure force, buoyancy force, virtual mass effect and Basset force to be very small. The lift forces in the present Stokes flow limit are negligible because of a lack of measurable particle spin (Magnus lift) and the laminar, low-level fluid shear fields (Saffman lift).

The motion of solid particles is suspension in a fluid is governed by the particle trajectory equation (Clift et al., 1978; Kleinstreuer, 2006) :

$$
m_{p} \frac{d^{2} \vec{x}_{p}}{d t^{2}}=C_{D P} \frac{\pi d_{p}^{2}}{4} \rho \frac{\left(\vec{v}-\vec{v}_{p}\right)\left|\vec{v}-\vec{v}_{p}\right|}{2}
$$

where $m_{p}$ is the mass of a single, spherical particle, $\vec{x}_{p}$ is the displacement, $\vec{v}$ is the fluid velocity, $\vec{v}_{p}$ is the particle velocity, and $C_{D P}$ is the drag coefficient (Clift et al., 1978).

The final equation may be stated as :

$$
S t \frac{d \vec{v}_{p}}{d t}=\left(\vec{v}-\vec{v}_{p}\right) \phi_{D} \frac{U}{D_{1}}
$$

where $\mathrm{U}$ is the mean inlet velocity, $D_{1}$ the parent duct diameter and $S t$ the Stokes number defined as :

$$
S t=\frac{\rho_{p} d_{p}^{2} U}{18 \mu D_{1}}
$$


where $\rho_{p}$ is the particle density and $\mu$ the fluid dynamic viscosity.

$\phi_{D}$ is a correction to Stokes' drag force for finite particle Reynolds numbers based on the slip velocity $\left(\vec{v}-\vec{v}_{p}\right)$ :

$$
\phi_{D}=C_{D p} \frac{R e_{p}}{24}
$$

where

$$
C_{D p}=\frac{C_{D}}{C_{\text {slip }}}
$$

where

$$
C_{D}= \begin{cases}\frac{24}{R e_{p}} & \text { for } \quad 0<R e_{p} \leq 1 \\ \frac{24}{R e_{p}^{0.646}} & \text { for } 1<R e_{p} \leq 400\end{cases}
$$

and the local particle Reynolds number is :

$$
R e_{p}=\frac{\rho d_{p}\left|\vec{v}-\vec{v}_{p}\right|}{\mu}
$$

The correlation for the slip factor, $C_{\text {slip }}$, is also given by Clift et al. [1], [23], [2] :

$$
C_{\text {slip }}=1+\frac{2 \lambda}{d_{p}}\left[1.142+0.058 \exp \left(-0.999 \frac{d_{p}}{2 \lambda}\right)\right]
$$

where $\lambda$ is the mean free path in fluid.

The representative Stokes number range [17] computed is [0.2-1.2].

The deposition efficiency, DE (\%), was defined as :

$$
D E=\frac{\text { particle number deposited in a given region }}{\text { total particle number entering that region }}
$$

\section{Results}

In this paper, we will only present the validation of the software on a 3 bifurcations Weibel model (fig. 1) as well as the particle deposition in the rat and the rabbit lungs for steady flow rate.

\subsection{Model validation}

The current simulation code (CFD-ACE) has been validated via comparisons with experimental data sets provided by Zhao and Lieber [26], (see Fig. 2). Steady flow was considered. For details of this particular case study, Comer et al. [3] and Zhang and Kleinstreuer [16].

For steady flow, a fully-developed, i.e., parabolic, velocity profile can be observed in both numerical and experimental studies at location 2 (Fig. 2(a)). At location 10 (see Fig. 2(c)), the velocity profile is skewed to the inner wall when the airflow turns from the parent to the daughter tube. When the airflow continuously turns, more 

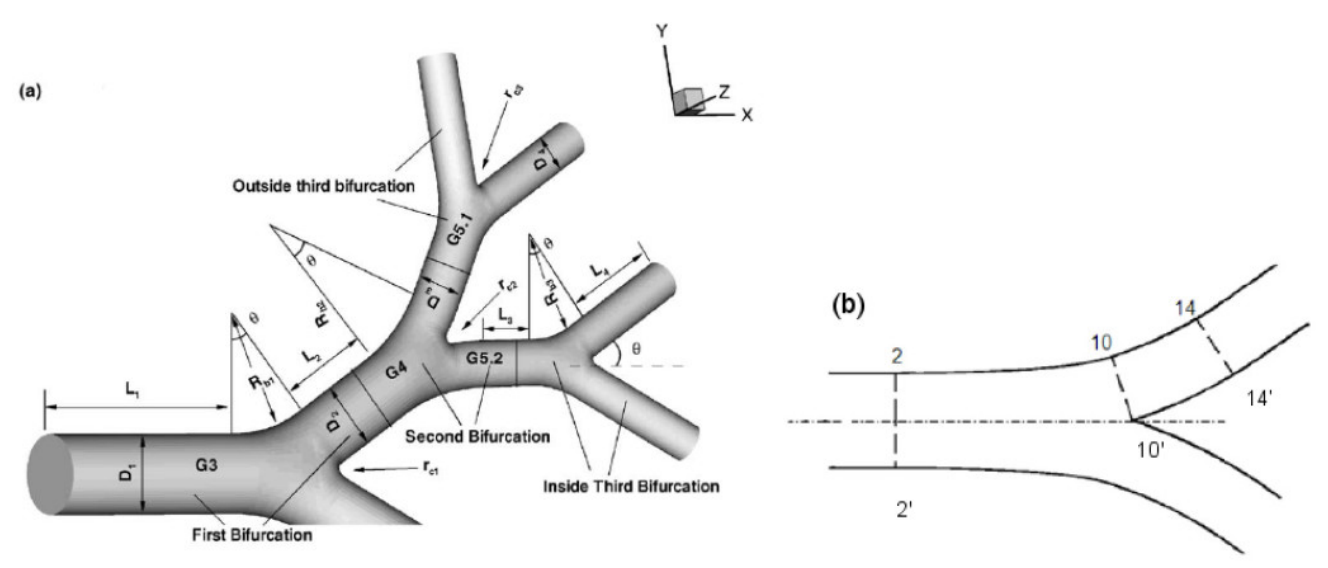

FiguRE 1 - Schematic of symmetric : a) triple bifurcation geometry [24]; b) single bifurcation of station positions [26],

fluid is pushed to the inner wall at location 14 (see Fig. 2(e)) due to the effects of a centrifugal force and secondary flow.

The airflow patterns in the lung airways are determined by the fluid property, the inlet condition and geometric characteristics. The geometric influence on the local airflow pattern is significant (see Figs. 2(b), (d) and (f)). The axial velocity is zero near the wall and maximum at the center of the airway (Fig. 2(b)).

Fig. 3 shows the comparison of our deposition efficiency in double bifurcation inplane model for steady flows with the data of Kim \& Fisher (1999) [14]. Due to the difficulty in strictly controlling the flow rate and aerosol diameter, the Reynolds numbers significantly varies during the experiments. Furthermore, some differences between computational predictions and experimental measurements may be caused by subtle discrepancies in model geometries and deposition measurements. Thus, when neglecting the experimental Reynolds number effect and focusing on deposition efficiency, the simulation results agree well with the experimental data of Kim \& Fisher.

The good agreement indicates that the CFD-ACE code is sufficiently accurate to simulate actual airflow and particle deposition in the human lung.

\subsection{The Rat configuration}

The rat geometry form medical imaging is exported in stereolithography format $S T L$ to be smoothed, repaired (holes geometry) and meshed with CFD-GEOM, the mesh generator of the CFD-ACE package. We generated several meshes - 300k to $4.3 \mathrm{M}$ cells - to check mesh convergence results. The breath of a rat of $500 \mathrm{~g}$ being of $4 \mathrm{ml} . \mathrm{s}^{-1}$, this gives us in our setup a inlet velocity of $1 \mathrm{~m} . \mathrm{s}^{-1}$ so a number Reynolds $R e_{D_{\text {inlet }}}=152$ which suggests that the flow is steady and laminar.

Figure 4 shows the pressure drop and the shape of the streamlines in the lungs of rats : the flow is relatively simple and does not appear to be circulating in the light of the low curvature bifurcations. The difficulty here doesn't clearly lie on the simulation itself but rather on the generation of a viable geometry and mesh : in this 

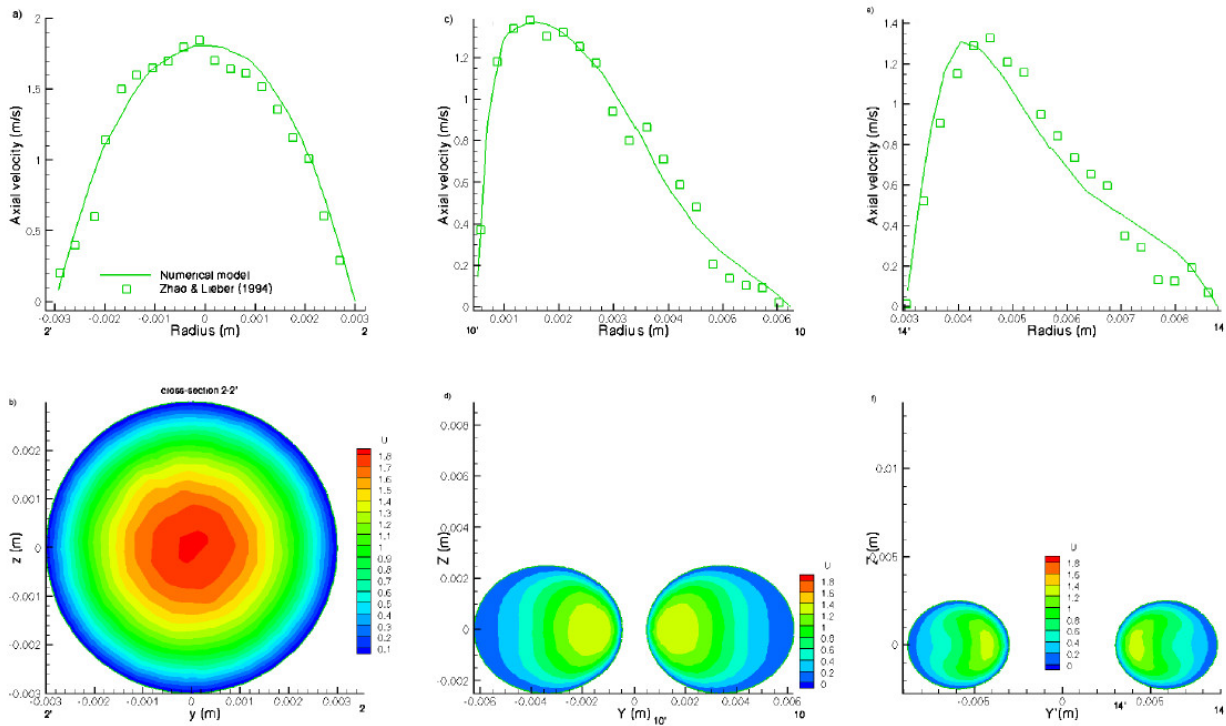

FiguRE 2 - Axial velocity distribution in steady flow : $\left(R e_{D 1}=1000\right)$; a) station $\left(2^{\prime}\right)-(2)$, c) station $\left(10^{\prime}\right)-(10)$ and e) station $\left(14^{\prime}\right)-(14)$ [26] (see Fig.1 b)) and flow patterns : b) axial velocity pattern at Slice $2, \mathrm{~d})$ at slice 10 and f) at slice 14 .
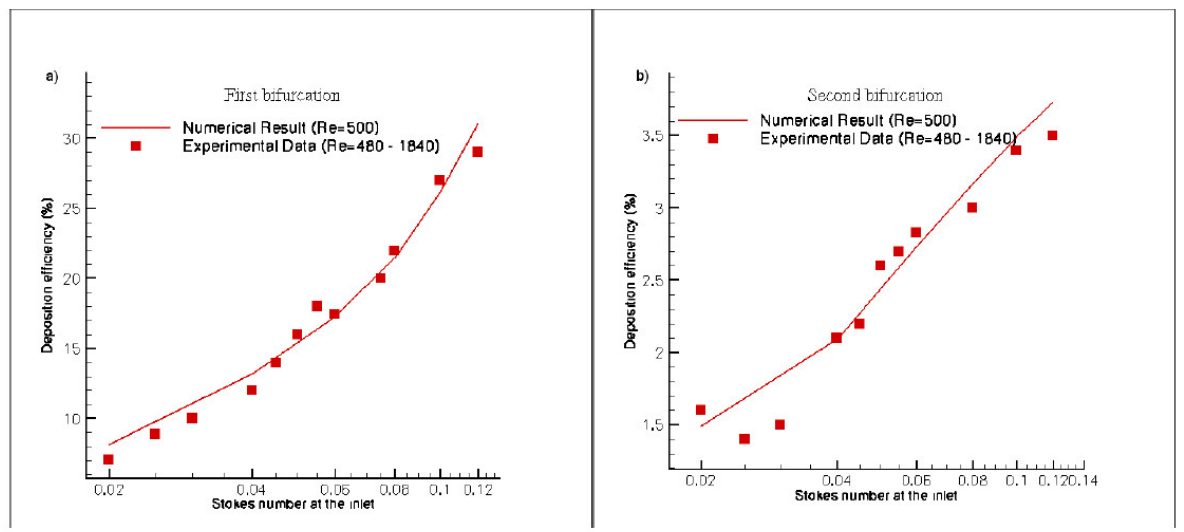

FIgURE 3 - Comparison of the numerical and experimental particle deposition efficiency (DE) in the double bifurcation in-plane model under steady flow (Experimental data were obtained from Kim \& Fisher, 1999 [14]) : a) DE at the first bifurcation; and DE at the second bifurcation. 
case, the initial geometry included non-physical constrictions on multiple outputs which made the simulation completely unrealistic even lengthening out through pipes. These constrictions have been smoothed the outlets extended with pipes.

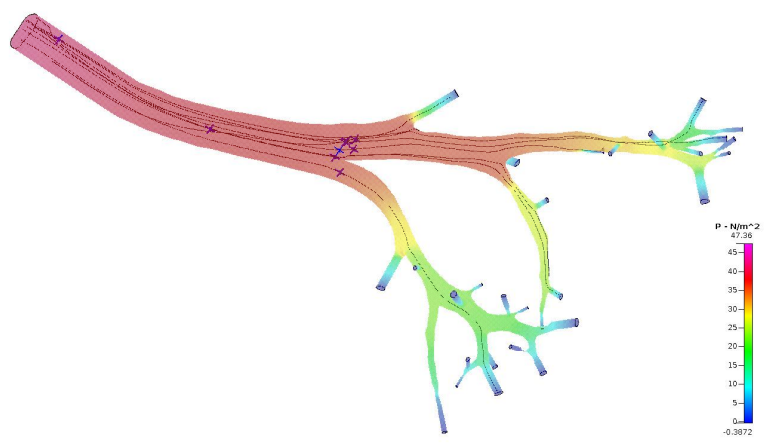

Figure 4 - Visualisation of the pressure drop and streamlines in rat lungs

Figure 5a) shows the deposition of particles observed. We note that the deposition appears only at junctions and bends which is very understandable because the geometry of the lungs of rats is fairly linear unlike human lungs and we had already observed that the deposition mechanism was an inertial mechanism. The results for the depositions were obtained with four intermediate meshes and the convergence of the results is obtained (Figure $5 \mathrm{~b}$ ) on the finest grid.
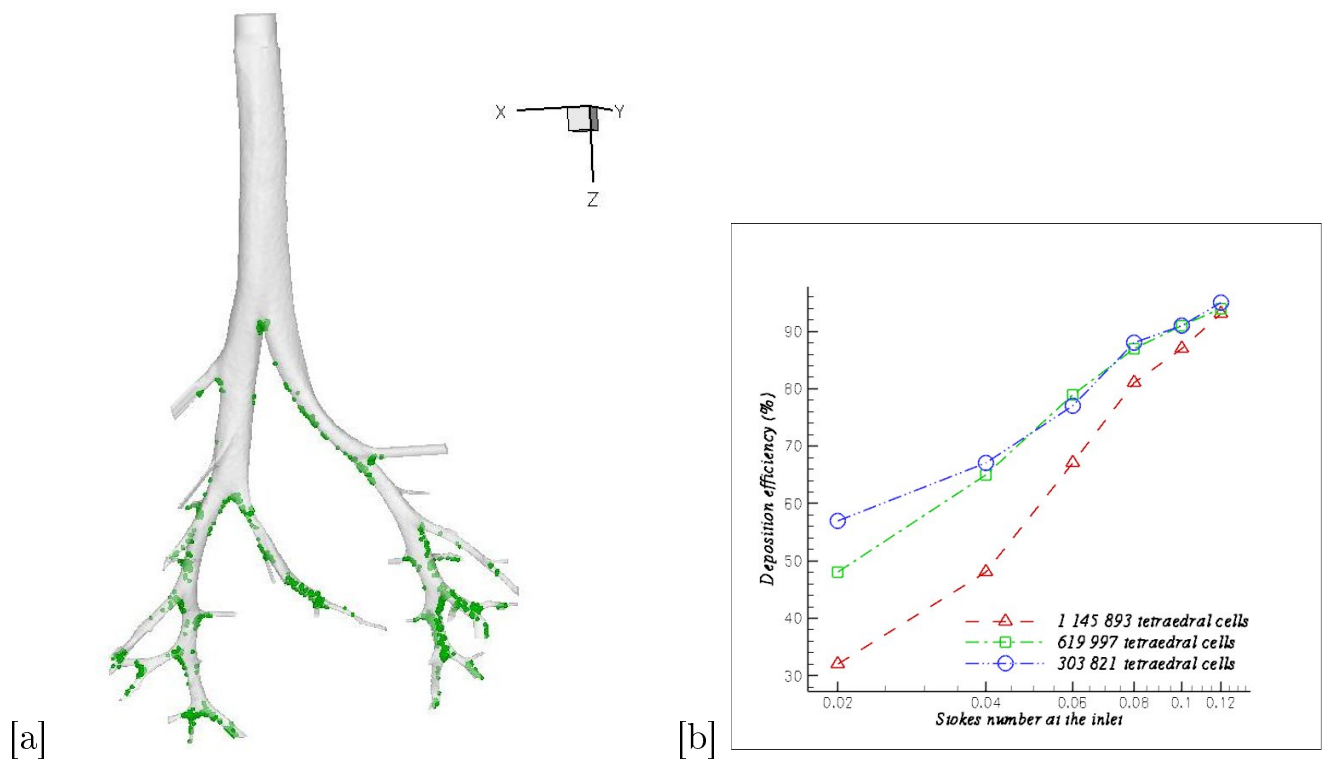

Figure 5 - a) Deposition efficiency and b) in rat lungs

\subsection{The Rabbit configuration}

Monash University, Australia has developed a technique the breath of an intubated rabbit and 4D images (3D over time) obtained by a CT scanner synchrotron which gives a very good resolution both spatially and temporally. We were able to get a 
geometry (Figure 6a) and we hardly generated meshes from $1.2 \mathrm{M}$ to $4.4 \mathrm{M}$ cells. In the simulations the inlet velocity was imposed at $1 \mathrm{~m} . \mathrm{s}^{-1}$ corresponding to a laminar Reynolds number of $R e=176$.
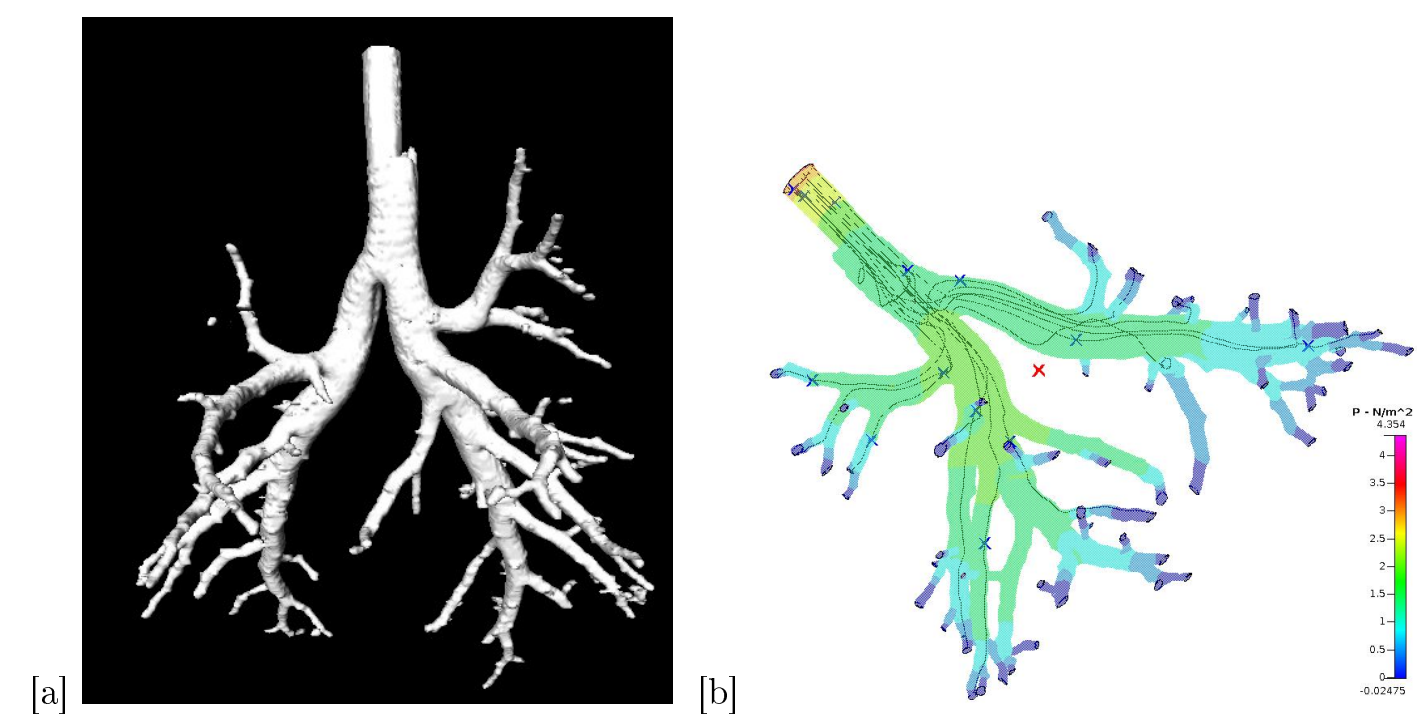

FiguRE 6 - a) Rabbit geometry from segmentation and b) Streamlines and pressure drop on a $4.4 \mathrm{M}$ cells grid

Figure $6 \mathrm{~b}$ ) shows the streamlines of the flow where you can note the existence of a vortical area downstream of the endotracheal area. The streamlines are pretty tortured in the first bifurcations which suggests that there is still recirculation zones. Here we find a similar behaviour to a flow in the presence of Dean vortices.

Regarding the deposition of particles, the same conclusion as with the Rat geometry can be dressed (Figure 7). This is mainly due to the fact that with our unstructured grid generation software, it's very difficult to increase the grid density near the walls without dramatically increasing the total number of cell. This is the reason why we started to develop a new software at ICUBE based on Immersed Boundary Methods, unstructured Cartesian cells and Automatic Grid Refinement.

\section{Conclusions}

After a validation phase, we have simulated the flow and the particle deposition in many Weibel's generic models and in anatomically realistic geometries of rat and rabbit coming from medical imaging. The particle deposition is mainly inertial and increases with the Stokes number and the Reynolds number. One of the major difficulty of this study is the generation of the appropriate mesh for the realistic geometries. 
[a]

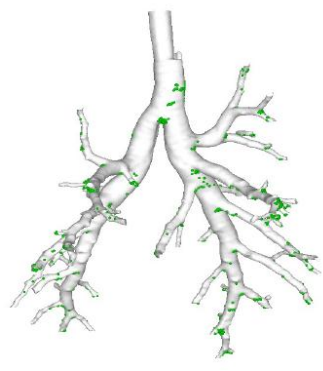

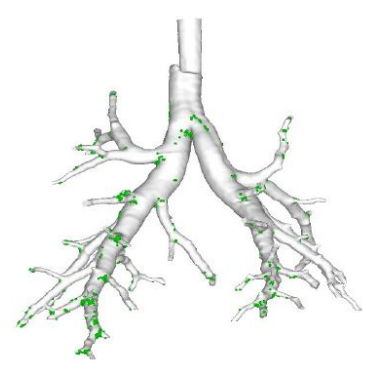

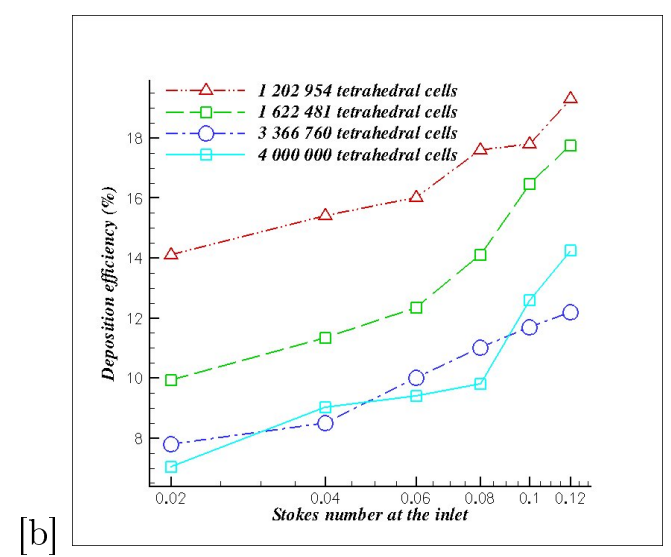

Figure 7 - a) Deposition and b) DE in Rabbit lungs

\section{Références}

[1] R. Clift, J. R. Grace, and M. E. Weber. Bubbles, drops, and particles. Academic Press, New York, 1978.

[2] J. K. Comer, C. Kleinstreuer, and C. S. Kim. Flow structures and particle deposition patterns in double-bifurcation airway models. part 2. aerosol transport and deposition. Journal of Fluid Mechanics, 435 :55-80, 2001.

[3] J. K. Comer, C. Kleinstreuer, and Z. Zhang. Flow structures and particle deposition patterns in double-bifurcation airway models. part 1. air flow fields. Journal of Fluid Mechanics, $435: 25-54,2001$.

[4] S. Dubsky, Hooper, S.B., K.K.W. Siu, and A. Fouras. Synchrotron-based dynamic computed tomography of tissue motion for regional lung function measurement. Journal of the Royal Society Interface, 9(74) :2213-2224, 2012.

[5] C. A. Dunbar and A. J. Hickey. Design of aerosol systems for drug delivery to the lungs using numerical methods. Medical applications of computer modelling : the respiratory systems WIT press Southampton, 2001.

[6] C. Ertbruggen, C. Hirsch, and M. Paiva. Anatomically based three-dimensional model of airways to simulate flow and particle transport using computational fluid dynamics. journal of Applied Physiology, 2004.

[7] A. Fouras, B.J. Allison, Dubsky Kitchen, M.J., J.T. S., Nguyen, K. Hourigan, K.K.W. Siu, K. Uesugi, N. Yagi, R.A. Lewis, M.J. Wallace, and S.B. Hooper. Altered lung motion is a sensitive indicator of regional lung disease. Annals of Biomedical Engineering, 40(5) :1160-1169, 2012.

[8] J. Hammersley and D. E. Olson. Physical models of the smaller pulmonary airways. J. Appl. Physio, 1992.

[9] J. Hegedus, I. Balashazy, and A. Farkas. Detailed mathematical description of the geometry of airway bifurcations. J. Appl. Physio, 2004.

[10] K. Horsfield and G. Cumming. Morphology of the bronchial tree in man. J. Appl. Physio, 1968.

[11] K. Horsfield, G. Dart, and D. E. Olson. Models of the human bronchial tree. J. Appl. Physio, 1971.

[12] H. Ilmi Robleh. Modélisation numérique des écoulements pulmonaires. PhD thesis, 2012.

[13] I. M. Katz, J. D. Schroeter, and T. B. Martonen. Factors affecting the deposition of aerosolized insulin. Diabetes Technology and Therapeutics, $3: 387-397,2001$. 
[14] C. S. Kim and D. M. Fisher. Deposition characteristics of aerosol particles in sequentially bifurcating airway models. Aerosol Science and Technology, 31 :198-220, 1999.

[15] Suki B. Kitaoka H, Takaki R. A three-dimensional model of the human airway tree. J Appl Physiol., 87(6) :2007-2017, 1999.

[16] Z. Li, C. Kleinstreuer, and Z. Zhang. Simulation of airflow fields and microparticle deposition in realistic human lung airway models. part 1 : Airflow patterns. European Journal of Mechanics B/Fluids, 26 :632-649, 2007.

[17] Z. Li, C. Kleinstreuer, and Z. Zhang. Simulation of airflow fields and microparticle deposition in realistic human lung airway models. part 2 : Particle transport and deposition. European Journal of Mechanics B/Fluids, 26 :650-668, 2007.

[18] T. Miki, Y. Imai, T. Ishikawa, and T. Yamaguchi. Airflow simulation of inspiration and expiration using a patient-specific model. Communication in BioEngineering 09, Oxford, England, 24-25 September 2009.

[19] N Nowak, Prashant P. Kakade, and Ananth V. Annapragada. Computational fluid dynamics simulation of airflow and aerosol deposition in human lungs. Annals of Biomedical Engineering, 2003.

[20] E. R. Weibel. Morphometry of the human lung. New York, Academic Press, 1963.

[21] E. R. Weibel. The pathway for oxygen, structure and function in the mammalian respiratory system. Harvard University Press, 1984.

[22] O. Witschger and J. F. Fabriès. Particules ultra-fines et santé au travail. 1- charactéristiques et effets potentiels sur la santé. INRS, Département Métrologie des polluants, Second trimestre, pages 21-35, 2005.

[23] Z. Zhang, C. Kleinstreuer, and C. S. Kim. Aerosol deposition efficiencies and upstream release position for different inhalation modes in an upper bronchial airway model. Aerosol Sciences and Technology, 36 :828-844, 2002.

[24] Z. Zhang, C. Kleinstreuer, and C. S. Kim. Cyclic micron-size particle inhalation and deposition in a triple bifurcation lung airway model. Journal of Aerosol Science, 33 :257-281, 2002.

[25] Y. Zhao and B. B. Lieber. Steady inspiratory flow in a model symmetric bifurcation. Trans. ASME : J. Biomech. Engrg., 116 :488-496, 1994.

[26] Y. Zhao and B. B. Lieber. Steady inspiratory flow in a model symmetric bifurcation. Trans. ASME : J. Biomech. Engrg., 116 :488-496, 1994. 